\title{
Processing aspects related to permanent GPS arrays
}

\author{
H. Bock ${ }^{1}$, G. Beutler ${ }^{1}$, S. Schaer ${ }^{1}$, T. A. Springer ${ }^{1}$, and M. Rothacher ${ }^{2}$ \\ ${ }^{1}$ Astronomical Institute, University of Berne, Switzerland \\ ${ }^{2}$ Institute for Astronomical and Physical Geodesy, Technical University of Munich, Germany
}

(Received December 20, 1999; Revised May 22, 2000; Accepted May 22, 2000)

\begin{abstract}
Preprocessing is an essential aspect for zero- and double-difference GPS software packages. In the first case we have to produce "clean" code and phase observations on the single receiver and single satellite level, in the second case solely double-difference observations have to be checked. The checks usually are performed on the "minimum constellation" level, i.e., the single receiver level for zero-, the single baseline level for double-difference packages. When analyzing the code observations stemming from a permanent array, this step may be performed in a much more efficient and robust way because the known geometry and, if available, the known atmospheric delays may be removed from the original observations. In an array of $n$ receivers observing $m$ satellites this leaves us with $n \cdot m$ observations and $n+m-1$ unknowns (the clock parameters relative to a reference clock). The degree of freedom of $f=n \cdot m-(n+m-1)$ (for, e.g., $n=10$ and $m=10$, we have $f=81$ ) allows for a very robust detection of outliers and enables generating a satellite clock file based on code measurements (with very much reduced multipath and noise characteristics). A similar step may be performed with the differences of phase observations between subsequent epochs. Using an analogous procedure as in the case of code observations we may generate phase files with all cycle slips flagged or, in the case of "small-area" arrays, even with cycle slips repaired. Both steps, phase and code cleaning, are performed in the same program unit. We discuss this new development and present first results and applications using data from the AGNES (Automated GPS Network Switzerland) and the IGS (International GPS Service) Networks.
\end{abstract}

\section{Introduction}

In the "old days", GPS preprocessing mainly dealt with data from one receiver or from one baseline. Checking GPS observations for bad data on the zero-difference level with one station is still a delicate issue and when processing baselines on the double-difference level we do not see where a data problem occurred.

Nowadays, many permanent GPS arrays are continuously operated, e.g., the IGS (International GPS Service). It makes sense to use the information stemming from these networks for novel and more effective preprocessing strategies. The proposed procedures are based on the idea to remove a priori known information from the original observations. This allows for a robust detection of outliers. Moreover, we may extract high quality high-rate satellite clocks in an efficient way. It is also possible to study the ionospheric conditions above local arrays.

\section{Theory and Developments}

Let us assume that we process all code and phase data of all stations of a permanent GPS array in an epoch-by-epoch mode.

Copy right(c) The Society of Geomagnetism and Earth, Planetary and Space Science (SGEPSS); The Seismological Society of Japan; The Volcanological Society of Japan; The Geodetic Society of Japan; The Japanese Society for Planetary Sciences.

\subsection{Code analysis}

The original GPS code observation equation (somewhat simplified and without error term) reads as:

$$
\begin{aligned}
p_{k}^{j}\left(t_{i}\right)= & \rho_{k}^{j}\left(t_{i}\right)-c \cdot \Delta t^{j}\left(t_{i}\right)+c \cdot \Delta t_{k}\left(t_{i}\right) \\
& +\Delta \rho_{k, \text { ion }}^{j}\left(t_{i}\right)+\Delta \rho_{k, \text { trop }}^{j}\left(t_{i}\right)
\end{aligned}
$$

with

$\begin{array}{ll}\text { the observed code pseudorange } & p_{k}^{j}, \\ \text { the geometrical distance } & \rho_{k}^{j}, \\ \text { the satellite clock correction } & c \cdot \Delta t^{j}, \\ \text { the receiver clock correction } & c \cdot \Delta t_{k}, \\ \text { the ionospheric refraction } & \Delta \rho_{k, \text { ion }}^{j}, \\ \text { the tropospheric refraction } & \Delta \rho_{k, \text { trop }}^{j},\end{array}$

where we use the subscripts $k$ to specify the receiver, the superscript $j$ to specify a satellite, and $t_{i}$ to specify the epoch.

Within a permanent network we usually know the geometry rather precisely. From the IGS, or one of its analysis centers, we may retrieve precise satellite orbits and possibly tropospheric zenith path delays. We may then remove the geometry $\rho_{k}^{j}$ and the tropospheric term $\Delta \rho_{k, \text { trop }}^{j}$ in Eq. (1). If the ionosphere-free linear combination (LC) of L1 and L2 is used, we are left with satellite and receiver clocks only:

$$
L C_{k}^{j}\left(t_{i}\right)=-c \cdot \Delta t^{j}\left(t_{i}\right)+c \cdot \Delta t_{k}\left(t_{i}\right),
$$

where $k$ is the receiver, $j$ the satellite index, and $t_{i}$ the epoch, and $L C_{k}^{j}$ is the ionosphere-free LC without geometry and tropospheric term. 
We may now perform epoch by epoch a satellite and receiver clock solution. One of the satellite or receiver clocks has to be chosen as reference clock and the degree of freedom is

$$
f=n \cdot m-(n+m-1)
$$

for, e.g., $n=10$ receivers and $m=10$ satellites, we have $f=81$.

\subsection{Phase difference analysis}

The original phase observation equation (somewhat simplified and without error term) reads as:

$$
\begin{aligned}
\phi_{k}^{j}\left(t_{i}\right)= & \rho_{k}^{j}\left(t_{i}\right)-c \cdot \Delta t^{j}\left(t_{i}\right)+c \cdot \Delta t_{k}\left(t_{i}\right) \\
& -\Delta \rho_{k, \text { ion }}^{j}\left(t_{i}\right)+\Delta \rho_{k, \text { trop }}^{j}\left(t_{i}\right) \\
& +\lambda \cdot N_{k}^{j}
\end{aligned}
$$

with

$\begin{array}{ll}\text { the observed phase pseudorange } & \phi_{k}^{j}, \\ \text { the geometrical distance } & \rho_{k}^{j}, \\ \text { the satellite clock correction } & c \cdot \Delta t^{j}, \\ \text { the receiver clock correction } & c \cdot \Delta t_{k}, \\ \text { the ionospheric refraction } & \Delta \rho_{k, i o n}^{j}, \\ \text { the tropospheric refraction } & \Delta \rho_{k, t r o p}^{j}, \\ \text { the initial integer ambiguity term } & \lambda \cdot N_{k}^{j},\end{array}$

where we use the subscripts $k$ to specify the receiver, the superscript $j$ to specify a satellite, and $t_{i}$ to specify the epoch.

We analyze differences of phase observations referring to subsequent epochs in order to eliminate the ambiguity term $\lambda \cdot N_{k}^{j}$. The elimination of the ambiguity term leads to an efficient procedure because we avoid the time consuming estimation of ambiguity terms. Apart from that we use the same procedure as in the case of code. Geometry, precise orbits, and troposphere contributions are removed and the ionosphere-free LC is used. The troposphere delays may be obtained either from troposphere estimates from IGS or from a troposphere model. For the code analysis the error made by using a model is negligible compared to the quality of the code measurements. For the phase analysis the model error only enters differentially and for a sampling rate of 30 seconds the effect is below $4 \mathrm{~mm}$ at an elevation cut off angle of $20^{\circ}$.

Based on these receiver and satellite clock respectively clock difference corrections we may clean the data by an iterative process. The residuals of the observations are checked and, if the absolute value of a residual exceeds a userspecified value, the observation is marked and excluded from the combination. We also check for each station whether both observation types are available for each satellite for the corresponding epochs and we count how many new ambiguities have to be set up during the entire observation period. At the end of the procedure we get a list of all marked observations and we may clearly identify stations with data problems.

Another important product of this data cleaning process are high-rate clock estimates for all satellites. "High-rate" means that we have one satellite clock correction for each observation epoch (normally 30 -second data, maybe 10 -second data in future). Clock corrections are estimated using code observations, clock difference corrections using phase difference observations. This procedure is very efficient. Multipath and noise characteristics are very much reduced by this robust clock estimation. We get in a simple way high-rate satellite clocks of good quality which in turn are useful for other applications. The larger the size of the permanent network the more satellites are observed simultaneously. In a global network the entire GPS constellation is "seen" simultaneously.

It makes sense to combine the clock corrections with code quality and the corresponding clock difference corrections based on the much better quality of phase observations into one set of satellite clock corrections of excellent quality to have only one clock set for the subsequent processing.

\subsection{Combined satellite clocks}

Let us consider one clock of a specific GPS satellite. For this satellite we have estimated one clock correction value $\widetilde{\Delta t_{i}}$ for each epoch $i=1, \ldots, n$ using the code observations (Eq. (2)). In the case of phase we have one clock difference correction value $\Delta \widetilde{\Delta t_{i, i+1}}$ for each epoch difference. We take these values as "pseudo-observations" for estimating a new combined clock correction set $\Delta t_{i}, i=1, \ldots, n$ (Eq. (5)). The observation equations for each epoch $i$ and each epoch difference read as:

$$
\begin{aligned}
& \text { Code }: \Delta t_{i}=\widetilde{\Delta t_{i}} \\
& \text { Phase }:-\Delta t_{i}+\Delta t_{i+1}=\Delta \widetilde{\Delta t_{i, i+1}} .
\end{aligned}
$$

Estimation of the combined clock turns out to be simple. The corresponding normal equation (NEQ) system has a (symmetric) tridiagonal structure. This NEQ system may be solved with a simple algorithm as described, e.g., in Press et al. (1992). The estimation may be performed independently for each satellite. The ratio of the weights of code clock values and phase clock difference values has to be chosen according to the RMS of code and phase measurements.

The resulting clock file may be used for

- studying the concept of virtual reference stations,

- preprocessing of observations of other stations,

- navigation, and for

- rapid and efficient LEO (Low Earth Orbiter) orbit determination.

\subsection{Ionosphere analysis}

The ionsphere analysis is also a useful processing aspect related to permanent networks. The procedure is similar to the phase difference analysis (Subsection 2.2). We use the plain difference between the L1 and L2 observations, the so-called geometry-free linear combination. The resulting signal is analyzed as a linear function (Eq. (6)) of the receiver latitude $(\beta)$ and longitude $(\lambda)$ (Schaer et al., 1999)

$$
\Delta \rho_{i o n}^{j}\left(t_{i}\right)=c_{0}+c_{\beta} \cdot \Delta \beta+c_{\lambda} \cdot \Delta \lambda,
$$

where $j$ is the satellite index and $t_{i}$ the epoch, $\Delta \rho_{i o n}^{j}$ is the observable, and $c_{0}, c_{\beta}$, and $c_{\lambda}$ are the model parameters established separately for each epoch and each satellite. Because of the fact that we use phase differences, the ionosphere development may only be studied relative to an initial epoch $t_{i}=t_{0}$.

\subsection{Spaceborne applications}

It is becoming routine to deploy spaceborne GPS receivers on LEOs. Such receivers may, e.g., be used for precise orbit 
Table 1. AGNES: Summary of station problems, August 19, 1998 ( $z$ : marked due to zenith distance, $c$ : refused due to code quality, $f$ : new ambiguity, $p$ : phase marked due to o-c, $m$ : phase-, but no code observations, $x$ : satellite not observed by enough stations).

\begin{tabular}{|l|rrrrrr|rc|}
\hline Site & \multicolumn{7}{|c|}{ \# Problems } & \multicolumn{2}{c|}{ \# Observations } \\
& $z$ & $c$ & $f$ & $p$ & $m$ & $x$ & Code & Phase \\
\hline EPFL & 0 & 0 & 92 & 1 & 1 & 0 & 24103 & 24103 \\
SIER & 0 & 0 & 66 & 4 & 1 & 0 & 20693 & 20693 \\
ZIMM & 0 & 0 & 87 & 1 & 0 & 0 & 23170 & 23170 \\
JUJO & 0 & 1 & 75 & 2 & 2 & 0 & 22677 & 22677 \\
FHBB & 0 & 0 & 88 & 6 & 0 & 0 & 24293 & 24293 \\
ETHZ & 0 & 0 & 86 & 3 & 0 & 0 & 23567 & 23567 \\
PFAN & 0 & 0 & 167 & 17 & 216 & 0 & 24177 & 24177 \\
\hline Total & 0 & 1 & 661 & 34 & 220 & 0 & 162680 & 162680 \\
\hline
\end{tabular}

determination (POD). We may use code and phase observations from such a GPS receiver to reconstruct the trajectory of the LEO. The high-rate satellite clocks derived from data of permanent arrays are very well suited for that purpose. We use them for single point positioning of the LEO using its code observations. Bad data may be recognized and eliminated. Furthermore we estimate position differences pertaining to subsequent epochs using phase observations using, e.g., the code-derived point positions as a priori information. The technique is also perfectly suited to detect cycle slips.

\section{Results}

\subsection{Local network: AGNES}

Figure 1 shows the station distribution of the AGNES network. The network serves several purposes, in particular monitoring and maintenance of the Swiss first-order control network (Landesvermessung), navigation, and research (GPS meteorology, geodynamics, etc.). The AGNES is deployed and operated by the Swiss Federal Office of Topography.

We processed one entire week of 30-second data of this permanent network to test our new procedures, Table 1 contains a summary of station problems for one day (August 19, 1998). It shows the outstanding quality of the AGNES data. In the example the maximum zenith distance was set to $z=90^{\circ}$, the maximum accepted absolute residual to $5 \mathrm{~m}$ for code, and $6 \mathrm{~mm}$ for phase observations, and the minimum number of stations observing one satellite to zero. In the column labeled $f$ we see the number of ambiguities set up for each station. A new ambiguity is set up if a cycle slip occurs (type " $p$ ") or if a satellite raises. There are not too many new ambiguities if we take into account that each of the 27 satellites passes once or even twice a day over one and the same region. The only receiver suffering some problems is Pfänder.

Figure 2 shows the estimated ionospheric gradient in longitude for one satellite as a result of the ionosphere analysis in the AGNES network. It is very consistent and far from being pure noise. The two curves correspond to an analysis using the entire AGNES, respectively the entire AGNES

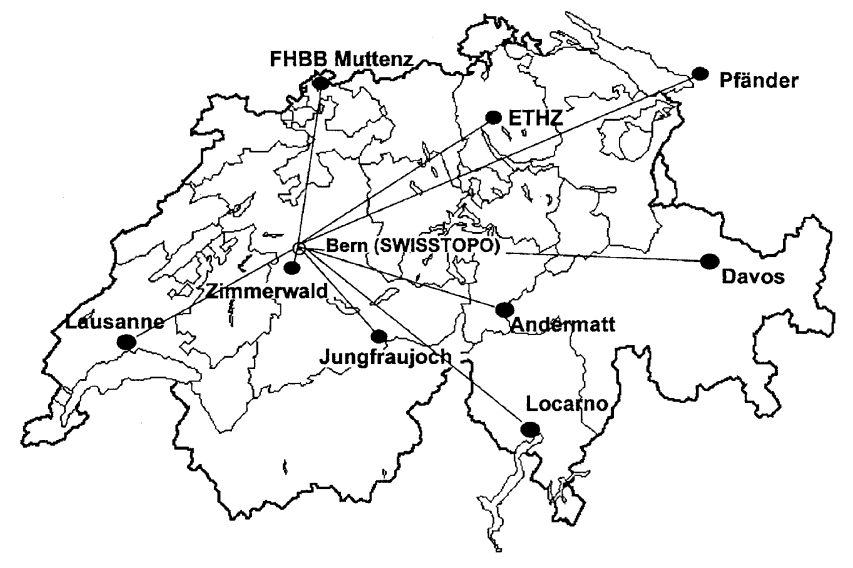

Fig. 1. The reference stations of the Swiss AGNES network in 1999.

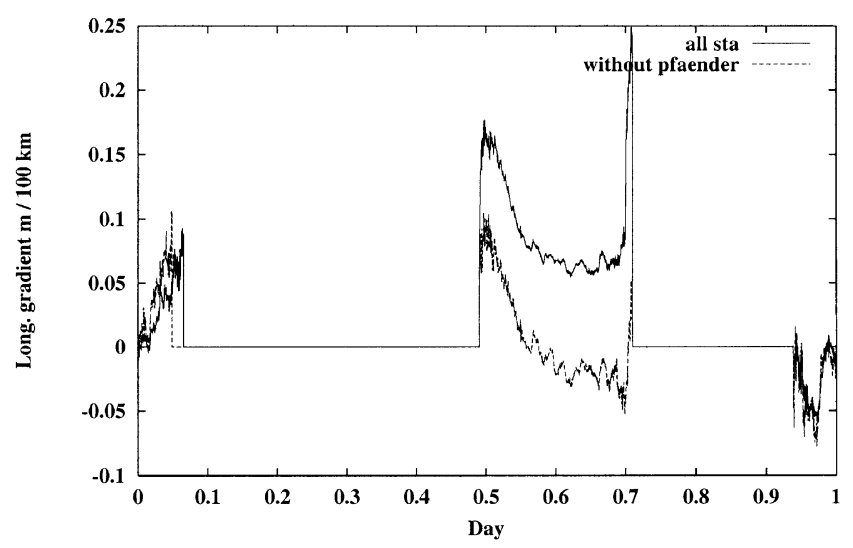

Fig. 2. Estimated ionospheric longitude gradient relative to first epoch, PRN 4.

except the Pfänder receiver. The estimates are quite consistent (4-15 mm RMS of observations) but the differences also underline that only part of the effect may be removed by such linear models (Eq. (6)) used in this case. The latitude gradient shows similar characteristics. This satellite-specific 


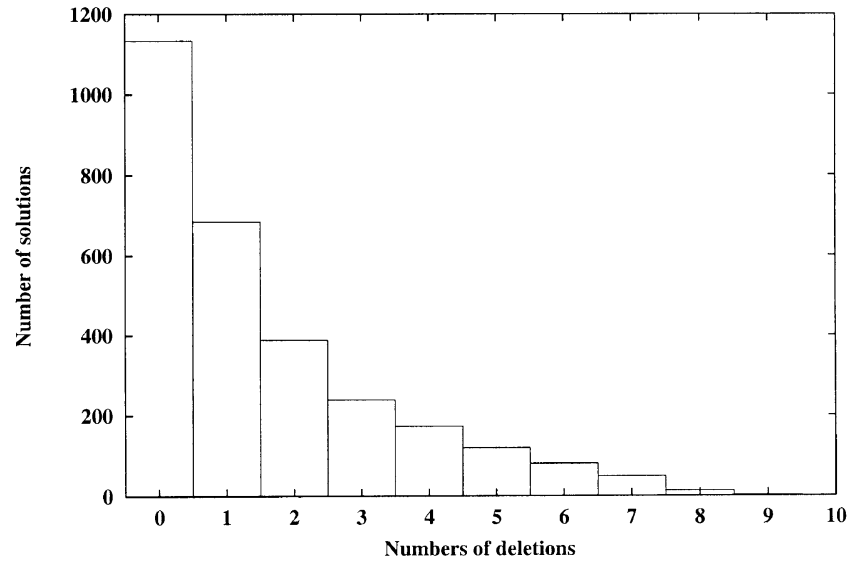

Fig. 3. Histogram of deleted code observations per epoch.

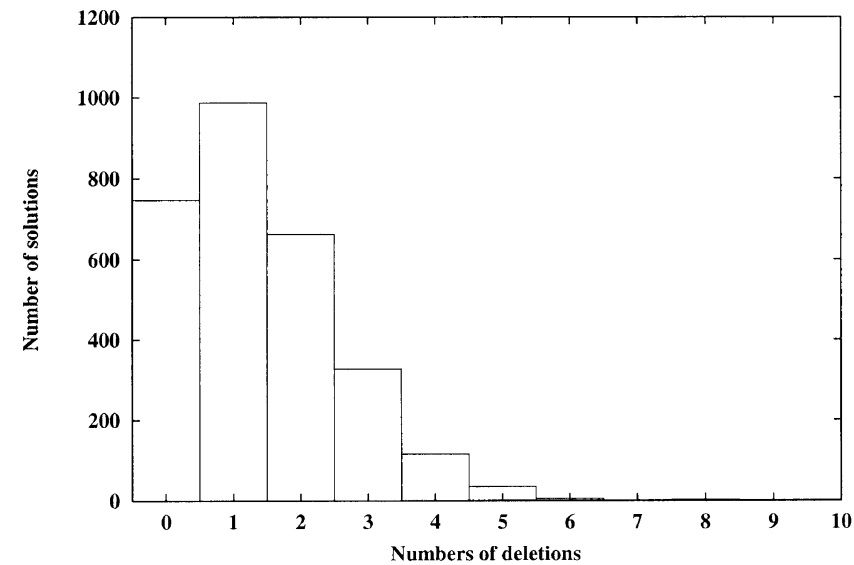

Fig. 4. Histogram of deleted phase observations per epoch.

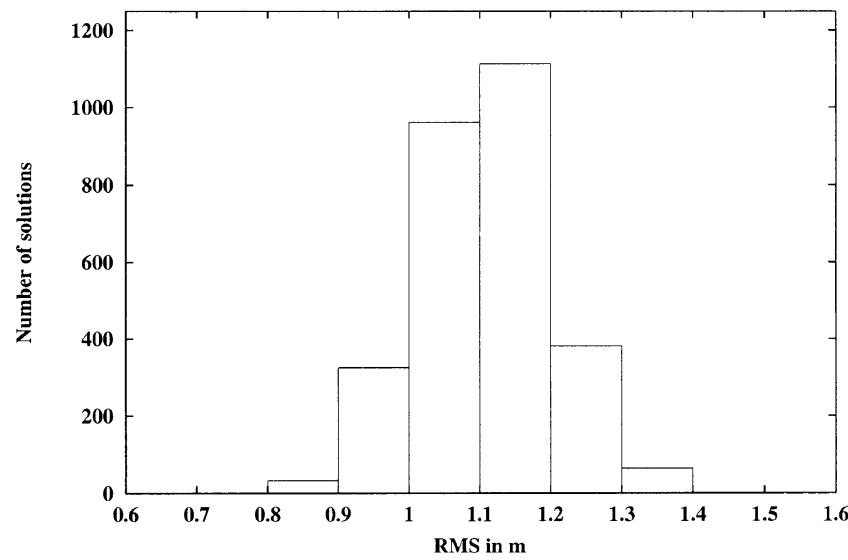

Fig. 5. Histogram of RMS of code clock correction estimation per epoch.

ionosphere analysis only makes sense for local and regional arrays.

\subsection{Global network: subset of the IGS network}

The IGS network is the largest global permanent GPS array. We may also use it to test our developments. We used data from June 29, 1995 and from March 30, 1996 and pro-
Table 2. RMS of code single point positioning using different satellite clocks.

\begin{tabular}{|l|ccc|}
\hline \multirow{2}{*}{ Site } & \multicolumn{3}{|c|}{ RMS (m) of CODSPP } \\
& JPL & CODE & NEWCLK \\
\hline KELY & 0.99 & 1.00 & 1.00 \\
KIRU & 1.23 & 1.26 & 1.25 \\
KIT3 & 1.18 & 1.15 & 1.27 \\
KOKB & 0.87 & 0.95 & 0.88 \\
KOSG & 0.76 & 0.81 & 0.82 \\
KWJ1 & 0.95 & 1.04 & 0.96 \\
USUD & 0.74 & 0.75 & 0.75 \\
POL2 & 0.57 & 0.62 & 0.60 \\
PERT & 0.68 & 0.66 & 0.78 \\
ONSA & 0.74 & 0.77 & 0.80 \\
MONP & 0.52 & 0.59 & 0.54 \\
JOZE & 0.82 & 0.83 & 0.89 \\
FORT & 0.79 & 0.78 & 0.80 \\
BOR1 & 0.71 & 0.75 & 0.81 \\
\hline
\end{tabular}

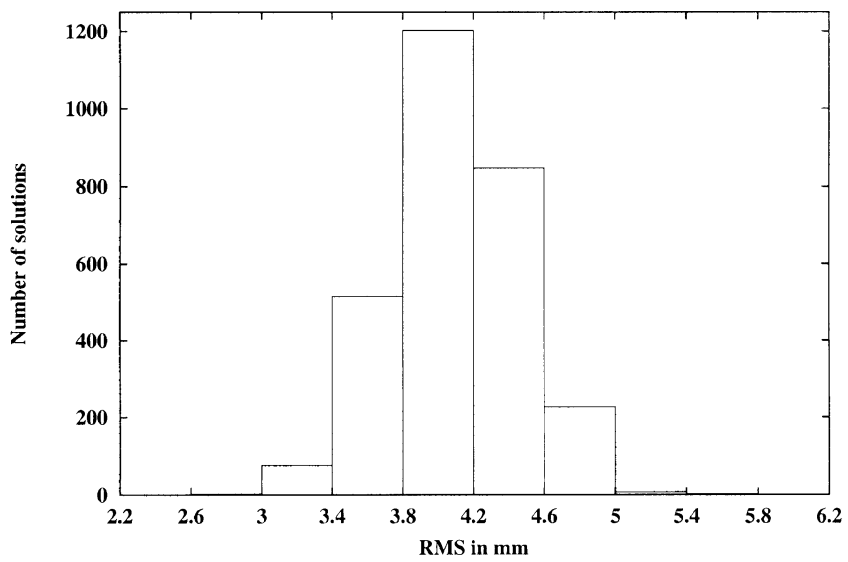

Fig. 6. Histogram of RMS of phase clock difference correction estimation per epoch difference.

cessed that part of the IGS network which was used in the CODE (Center for Orbit Determination in Europe) routine processing, using approximately 65 stations. Figures 3 and 4 show the number of deletions for code respectively phase observations for each epoch of March 30, 1996. The maximum number of deletions is 9 which is a remarkable good performance for a network of 65 stations.

The satellite clock file emerging from this processing step is complete, i.e., it has for each satellite and for each epoch a clock correction value because each satellite is observed at each epoch by at least one station of the global network. We thus obtain high-rate satellite clocks in a very efficient way and they are, in particular on the code basis, of comparable quality as the routine satellite clock estimates from JPL (Jet Propulsion Laboratory) or CODE. This comparison is docu- 


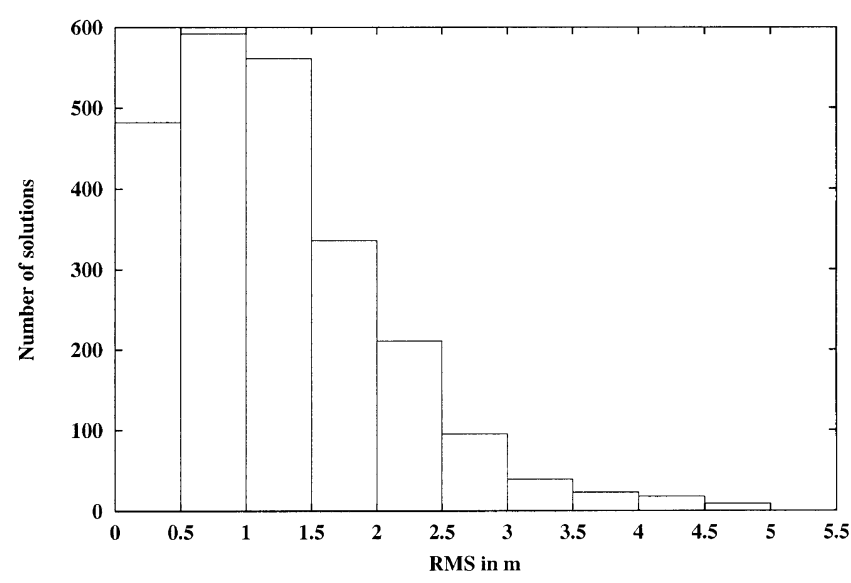

Fig. 7. Histogram of RMS of GPS/MET code single point positioning, March 30, 1996.

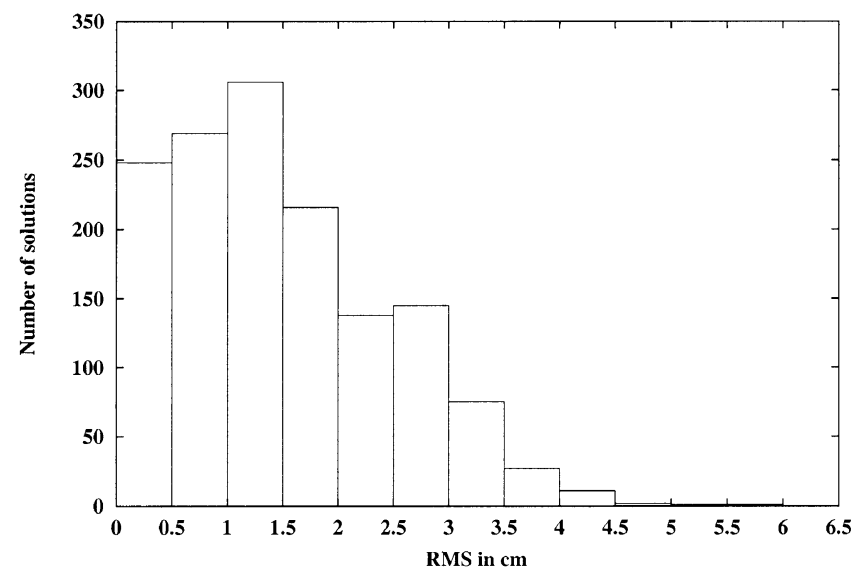

Fig. 8. Histogram of RMS of GPS/MET phase position differences, March $30,1996$.

mented in Table 2. We performed a single point positioning of a few stations using code observations using either precise clocks from JPL, CODE, or the new combined clocks derived by the method outlined in Subsection 2.3. Table 2 shows the RMS for the single point positioning using 5-minute data sampling for each station. The results are promising. One should in particular be aware of the fact that for March 30, 1996 clocks from the IGS and its analysis centers are only available with a sampling rate of 5 minutes whereas our highrate satellite clocks are available every 30 seconds. Let us point out again that our procedure is simple, efficient, and robust. We are able to estimate for each epoch the RMS of the residuals. If we inspect these RMS values of the code clock correction estimation (Fig. 5), we see that it is very consistent and at a level of about $1.1 \mathrm{~m}$. The RMS for each epoch difference of the clock correction differences based on phase observations (Fig. 6) is much lower at a level of about $4 \mathrm{~mm}$.

\subsection{Application to LEO orbit determination}

We gained first experiences with processing GPS data of a LEO by performing a single point positioning procedure for the LEO. For this purpose we used our clock estimates

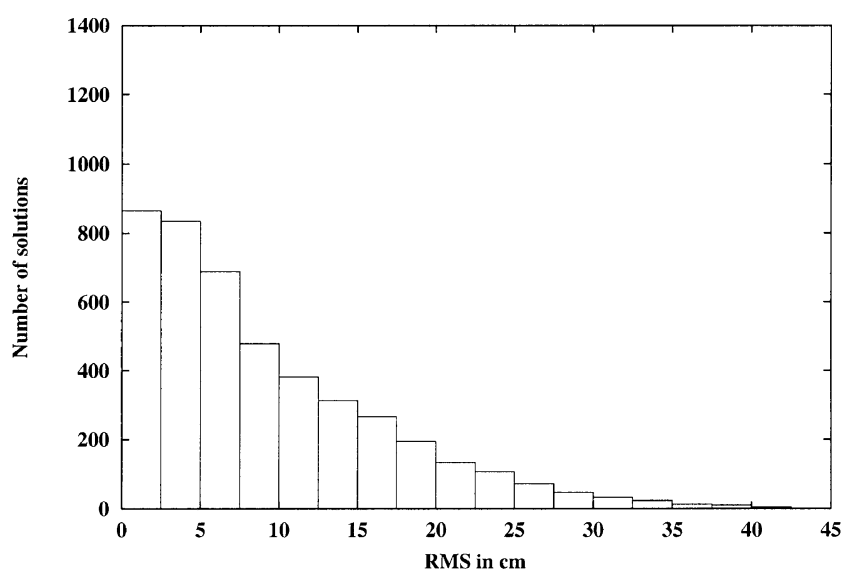

Fig. 9. Histogram of RMS of GPS/MET phase position differences using 10 -second data and quadratic satellite clock interpolation.

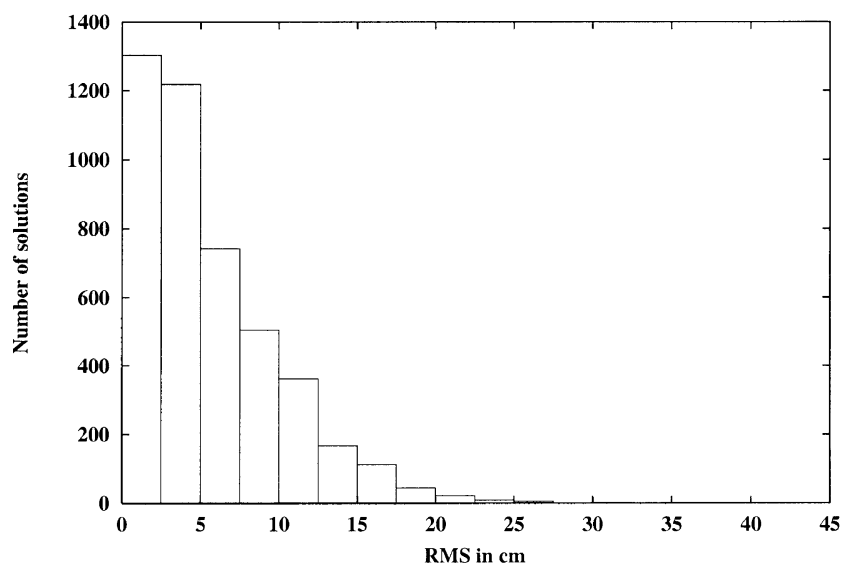

Fig. 10. Histogram of RMS of GPS/MET phase position differences using 10 -second data and cubic satellite clock interpolation.

based on the IGS network. We processed data of GPS/MET, a LEO in a circular orbit about $750 \mathrm{~km}$ above the surface of the Earth (Feng et al., 1995).

For the first results we sampled the 10 -second data and used only 30 -second data to have the same epochs as our highrate satellite clocks. When processing data of GPS/MET one experiences the problem that there are many epochs with only few good observations. This is due to the fact that the GPS antenna axis is mounted in a horizontal plane on the rear side of the satellite. This is not ideal for the determination of the position of GPS/MET because the number of visible GPS satellites is relatively low. Moreover we experience the problem that the signal of some GPS satellites may pass through low atmospheric layers. Such observations are of course useful for atmospheric studies (the primary goal of GPS/MET), they are not useful for POD (precise orbit determination). If we exclude these low elevation observations we get an accuracy of the single point positioning using code observations as it is shown in Fig. 7. We see that most values are below 2 to $2.5 \mathrm{~m}$ using the ionosphere-free linear combination. Furthermore, we determined position differences using differences of phase observations between subsequent epochs. Figure 8 
shows the RMS of these position differences using phase observations after excluding the low elevation observations. Mainly in processing the phase observations we notice that in some cases there are only four observations (four visible GPS satellites) available for determining the position differences, in which case we cannot estimate an RMS.

We also processed the full rate 10 -second data of the GPS/MET for single point positioning. In this case we had to interpolate the satellite clocks, however. At first we chose a quadratic interpolation between the given values (Fig. 9). The accuracy of the position differences computed with the interpolated values has deteriorated because the quadratic interpolation is not sufficient to model the S/A-effect over a time interval of 1.5 minutes. If we use a cubic interpolation it looks already better (Fig. 10). This result is rather interesting because it indicates that it might be possible to significantly reduce the S/A-effects using polynomial interpolation of a higher degree. We believe that a good single point positioning of LEOs with 10-second data should be possible. Based on our experiments we believe that 10 -second clocks from the global network and cubic interpolation would completely "remove" the influence of S/A.

\section{Summary and Outlook}

We have shown that we may easily recognize data problems on the zero-difference level of stations of a permanent GPS network using our newly developed algorithm. The algorithm is also applicable for networks where no troposphere parameters are available because it is sufficient to use a tropospheric model to clean the data. The generalization of the procedure into a receiver performance and ionosphere monitoring program will be the next step. Furthermore, we have shown that the high-rate satellite clock estimates as a product of this data cleaning process are of very good quality. The procedure is efficient and robust. For single point positioning of a LEO these high-rate satellite clocks are very well suited. We made first experiences with processing data stemming from the GPS/MET experiment. For precise orbit determination of the LEO the accuracy of the code-derived single point positions is not sufficient. The accuracy of the position differences derived from phase data, however, promises better point positions if we combine them with the code positions in the same way as the code clock corrections with the phase clock difference corrections (Subsection 2.3). In addition, we plan to enhance our force models for LEOs to reconstruct the trajectory at the few $\mathrm{cm}$ level.
We also consider generating code and phase observations for a virtual reference station. Such virtual reference stations are very useful for local and regional GPS arrays (Van der Marel et al., 1999). We believe that a special virtual reference station in the geocenter might be a useful tool for global permanent GPS networks. We view it as the double-differences equivalent of the precise point position technique (Zumberge et al., 1997).

Acknowledgments. The GPS/MET project is a proof-of-concept space mission to utilize GPS signals to determine atmospheric profiles. The data of GPS/MET have been made available to us through UCAR (University Coperation for Atmospheric Research), Boulder, U.S.A. These contributions are gratefully acknowledged.

\section{References}

Beutler, G., W. Gurtner, M. Rothacher, U. Wild, and E. Frei, Relative static positioning with the Global Positioning System: Basic Technical Considerations, in Global Positioning System: An Overview, Convened and Edited by Y. Bock and N. Leppard, Vol. 102, pp. 1-23, Springer-Verlag, Berlin Heidelberg, 1990.

Beutler, G., M. Rothacher, S. Schaer, T. A. Springer, J. Kouba, and R. E. Neilan, The International GPS Service (IGS): An Interdisciplinary Service in Support of Earth Sciences, Adv. Space Res., 23(4), 631-653, 1999.

Beutler, G., S. Schaer, and M. Rothacher, Wide Area Differential GPS, Internal Report, Printing Office, University of Berne, 1999.

Feng, D., B. Herman, M. Exner, W. Schreiner, R. McCloskey, and D. Hunt, Preliminary Results from the GPS/MET Atmospheric Remote Sensing Experiment, in GPS Trends in Precise Terrestrial, Airborne, and Spaceborne Applications, Convened and Edited by Beutler, Hein, Melbourne, Seeber, Vol. 115, pp. 139-143, Springer-Verlag, Berlin Heidelberg, 1995.

Press, W. H., S. H. Teukolsky, W. T. Vetterling, and B. P. Flannery, Numerical Recipes in Fortran 77, Second Edition, pp. 42f, Cambridge University Press, 1992.

Rothacher, M. and L. Mervart, Bernese GPS Software, Version 4.0, Astronomical Institute, University of Berne, 1996.

Schaer, S., G. Beutler, M. Rothacher, E. Brockmann, A. Wiget, and U. Wild, The Impact of the Atmosphere and Other Systematic Errors on Permanent GPS Networks, in Geodesy Beyond 2000-The Challenge of the First Decade, edited by K.-P. Schwarz, IAG Symposia, Vol. 121, pp. 373-380, Springer-Verlag, Berlin Heidelberg, 2000.

Van der Marel, H. and C. D. de Jong, Active GPS Reference System for the Netherlands, Allgemeine Vermessungsnachrichten, 2000 (in press).

Wanninger, L., Der Einfluss ionosphärischer Störungen auf die präzise GPSPositionierung mit Hilfe virtueller Referenzstationen, Zeitschrift für Vermessungswesen, 10(3), 322-331, 1999.

Zumberge, J. F., M. B. Heflin, D. C. Jefferson, M. M. Watkins, and F. H. Webb, Precise point positioning for the efficient and robust analysis of GPS data from large networks, J. Geophys. Res., 102(B3), 5005-5017, 1997.

H. Bock (e-mail: bock@aiub.unibe.ch), G. Beutler, S. Schaer, T. A. Springer, and M. Rothacher 